\title{
Experimental observation of true left-handed transmission peaks in metamaterials
}

\author{
Koray Aydin and Kaan Guven \\ Department of Physics, Bilkent University, Bilkent 06800, Ankara, Turkey \\ Maria Kafesaki \\ Research Center of Crete and University of Crete, Heraklion, Crete, Greece
}

Lei Zhang and Costas M. Soukoulis

Ames Laboratory, U.S. Department of Energy, and Department of Physics and Astronomy, Iowa State University, Ames, Iowa 50011

\section{Ekmel Ozbay}

Nanotechnology Research Center and Department of Physics, Bilkent University, Bilkent 0680o, Ankara, Turkey

Received June 29, 2004

\begin{abstract}
We report true left-handed ( $\mathrm{LH}$ ) behavior in a composite metamaterial consisting of a periodically arranged split ring resonator (SRR) and wire structures. We demonstrate the magnetic resonance of the SRR structure by comparing the transmission spectra of SRRs with those of closed SRRs. We have confirmed experimentally that the effective plasma frequency of the LH material composed of SRRs and wires is lower than the plasma frequency of the wires. A well-defined LH transmission band with a peak value of $-1.2 \mathrm{~dB}(-0.3 \mathrm{~dB} / \mathrm{cm})$ was obtained. The experimental results agree extremely well with the theoretical calculations. (C) 2004 Optical Society of America

OCIS codes: $120.7000,260.2110$.
\end{abstract}

In 1968, Veselago predicted that a medium with negative permittivity $\epsilon$ and negative permeability $\mu$ will exhibit unusual physical properties such as negative refraction, reversal of Doppler shift, and backward Čerenkov radiation. ${ }^{1}$ In such a medium the electric, magnetic, and wave vector components form a left-handed coordinate system; hence the name left-handed material is used for description. Recently this idea was experimentally investigated by construction of a composite metamaterial (CMM) consisting of two components that have $\epsilon(\omega)<0$ and $\mu(\omega)<0$ simultaneously over a certain frequency range. ${ }^{2,3}$ Whereas a medium with $\epsilon<0$ can easily be achieved, e.g., by periodically arranged metallic wires, ${ }^{4}$ obtaining $\mu(\omega)<0$ was a challenge because of the lack of a magnetic charge. Pendry et al. suggested that a periodic array of metallic split ring resonator (SRR) structures exhibits $\mu(\omega)<0$ close to magnetic resonance frequency $\omega_{p}{ }^{5}$ Various studies that employed different structure designs extended this investigation..$^{2,3,6-8}$ Negative refraction of electromagnetic waves at the interface of CMMs was also observed, ${ }^{9-11}$ which supports the existence of a left-handed material.

The existence of passbands for CMM within the respective stop bands of SRR-only and wire-only media is intuitively considered evidence of left-handed behavior. In this Letter, by measuring $\omega_{p}$ for the CMM and for wire-only structures, we demonstrate experimentally that the dielectric response of the CMM differs substantially from that of the wire-only medium. It is evident that a shift in plasma frequency renders the aforementioned intuitive approach inapplicable. This is a new attribute that was not recognized in previous research. Based on this observation, we present a new CMM structure that exhibits true left-handed behavior and has a transmission band with a peak value at $-1.2 \mathrm{~dB}$. To our knowledge, this is the highest transmission value reported for a left-handed material.

In general, a SRR structure exhibits both magnetic resonance ${ }^{5}$ induced by the splits at the rings and electric resonance ${ }^{12}$ induced by the dipolelike charge distribution along the incident electric field. A bandgap in the transmission spectrum of a periodic SRR medium may be due to negative $\epsilon$ or negative $\mu$ or solely to the periodicity. The ambiguity can be lifted by use of a structure in which the splits in the ring resonators are closed. This will destroy the magnetic resonance but maintain the electric resonance. A frequency gap that is present in the transmission spectrum of the SRR medium but not in that of the closed split ring resonator (CSRR) medium will then correspond to $\mu<0$.

The SRR [Fig. 1(a)] and CSRR [Fig. 1(b)] units are fabricated on a FR4 circuit board with a deposited copper layer of $30-\mu \mathrm{m}$ thickness. ${ }^{6}$ The geometric parameters of the SRR are $d=t=0.2 \mathrm{~mm}, w=0.9 \mathrm{~mm}$, and $r=1.6 \mathrm{~mm}$, as shown in Fig. 1(a). The circuit board has a 1.6-mm thickness and dielectric constant $\epsilon=4.4$. SRR units are arranged periodically, with 5,15 , and 18 unit cells in the $x, y$, and $z$ directions, respectively. The dimensions of a unit cell containing a single SRR are $a_{x}=a_{y}=8.8 \mathrm{~mm}$ and $a_{z}=6.5 \mathrm{~mm}$. Transmission measurements are performed in free space, where, unlike in a waveguide measurement, no restriction on 

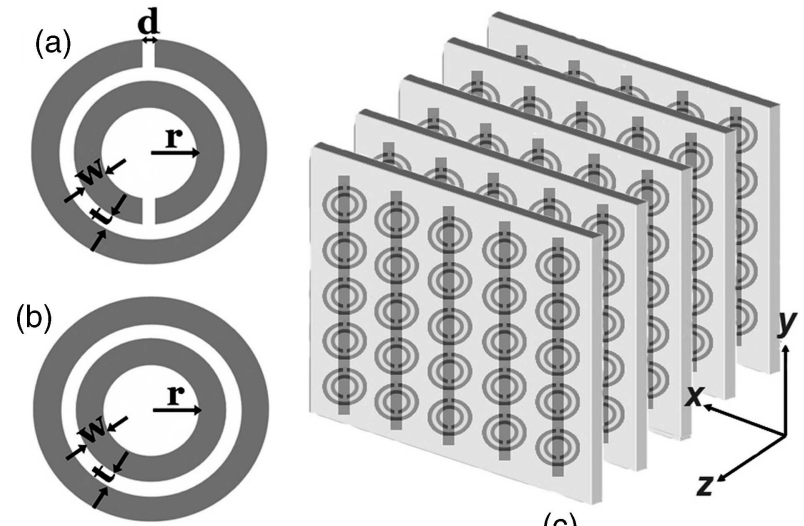

(c)

Fig. 1. Schematics of (a) a single SRR, (b) a CSRR, (c) a periodic CMM composed of SRRs on one side and wires on the other side of a dielectric board.

the size of the structures is imposed. The experimental measurement setup consists of a HP 8510C network analyzer and a set of microwave horn antennas for different parts of the frequency spectrum. The incident field propagates along the $x$ direction, with $\mathbf{E}$ and $\mathbf{H}$ along the $y$ and $z$ directions, respectively.

Figure 2 shows the measured transmission spectra of periodic SRRs and CSRRs from 3 to $14 \mathrm{GHz}$. The first bandgap (3.55-4.05 GHz) of the SRR medium is not present in the CSRR medium, indicating that $\mu<$ 0 . The second bandgap $(8.1-11.9 \mathrm{GHz})$ is present for both the SRR medium and the CSRR medium. This measurement clearly shows that the stop bands of a SRR medium cannot be automatically assumed to be negative- $\mu$ behavior. Some of the observed gaps could also originate from the electrical response of the SRRs or from Bragg gaps that are due to periodicity.

Another point to be discussed is the electric response of the CMM. Previously reported transmission results did not emphasize the contribution of the electric response of SRRs to that of wire structures. However, it was recently found ${ }^{12}$ that the SRRs, in addition to their resonant magnetic response at $\omega_{m}$, exhibit a resonant response at a higher frequency $\omega_{o}$ that has an electric character. This behavior is similar to that of a periodic cut-wire medium that exhibits a stop band with a well-defined lower edge that is due to the discontinuous wire geometry. ${ }^{8}$ As a result, the SRRs contribute to the effective permittivity of the CMM, causing a downward shift in the plasma frequency determined solely from wire structures. ${ }^{12}$ To demonstrate this effect we used a CMM consisting of periodic alternating layers of CSRRs and wires. The thickness, length, and width of the wires were $30 \mu \mathrm{m}$, $13.5 \mathrm{~cm}$, and $0.9 \mathrm{~mm}$, respectively. Figure 3 displays the measured transmission spectra of wire-only media and CMMs consisting of CSRR and wire layers. The $\omega_{p}$ of the wire-only structure near $8 \mathrm{GHz}$ is reduced to $5.3 \mathrm{GHz}$ within the closed CMM structure. As shown in Fig. 3, $\omega_{p}$ of the CMM is lower than that of the wire-only medium alone. It is crucial to determine whether the shift in plasma cutoff frequency covers the magnetic resonance gap, which would render the CMM a right-handed medium.
We have designed the present SRRs such that the first bandgap of a SRR structure at $3.55-4.05 \mathrm{GHz}$ is not obscured by this shift. The CMM structure is made from $N_{x}=5, N_{y}=15$, and $N_{z}=24$ unit cells. Each unit cell has a single SRR and a copper wire that comprises stacked SRR and wire layers [Fig. 1(c)], with lattice spacings $a_{x}=a_{y}=8.8 \mathrm{~mm}$ and $a_{z}=6.5 \mathrm{~mm}$. The transmission spectra for SRR only, wire only, and CMM periodic structures are displayed in Fig. 4(a). The simulation results [Fig. 4(b)] agree well with the experimental data.

The CMM structure permits propagation of electromagnetic waves at $3.6-4.1 \mathrm{GHz}$, where both $\epsilon$ and $\mu$ are negative. The CMM passband exactly coincides with the stop band of the SRR. The transmission peak at $3.9 \mathrm{GHz}$ is $-1.2 \mathrm{~dB}$, which is a significantly high value for a material made from metals. Similarly, the simulations predict a transmission peak at $3.92 \mathrm{GHz}$ of $-1.5 \mathrm{~dB}$. We stress that a similar transmission band is not present for a CMM composed of CSRRs and wires (Fig. 3). The electric response contribution of SRRs is also evident here: If $\omega_{p}$ of the wire-only structure [dashed curve in Fig. 4(a)] were used to identify the $\epsilon<0$ regime for the CMM, the transmission from 5.3 to $8 \mathrm{GHz}$ would have occurred in a regime with $\epsilon<0$ and $\mu>0$, which is not possible.

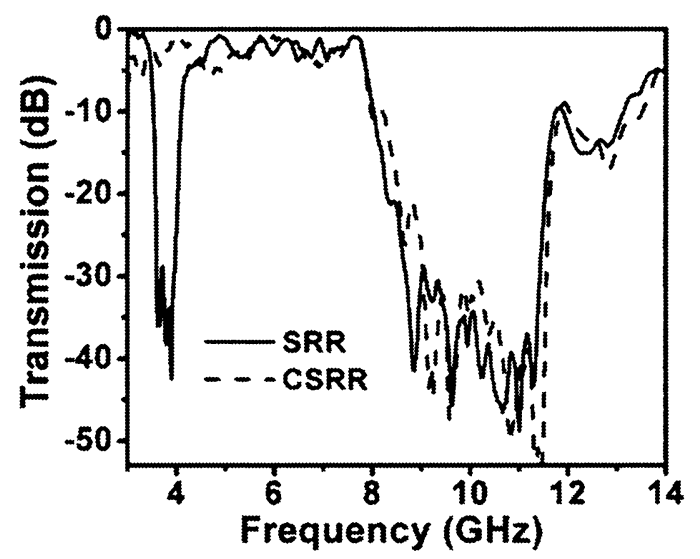

Fig. 2. Measured transmission spectra of a periodic SRR medium and a periodic CSRR medium from 3 to $14 \mathrm{GHz}$.

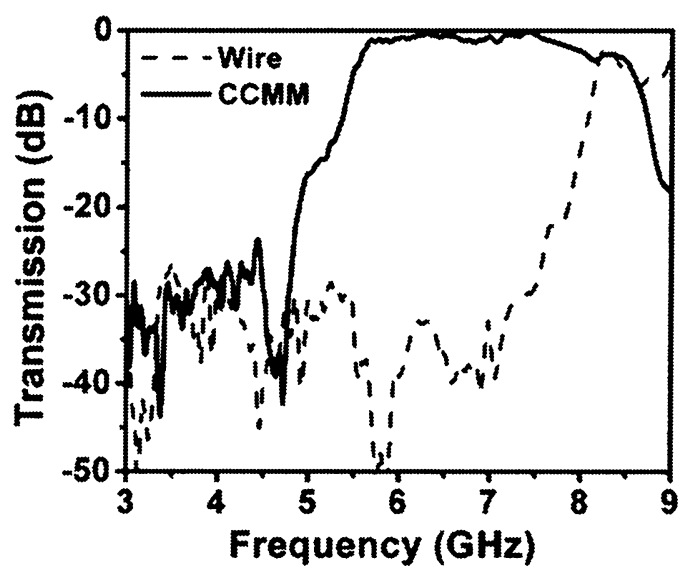

Fig. 3. Measured transmission spectra of wires and closed CMMs (CCMMs) achieved by periodic arrangement of CSRRs and wires. 

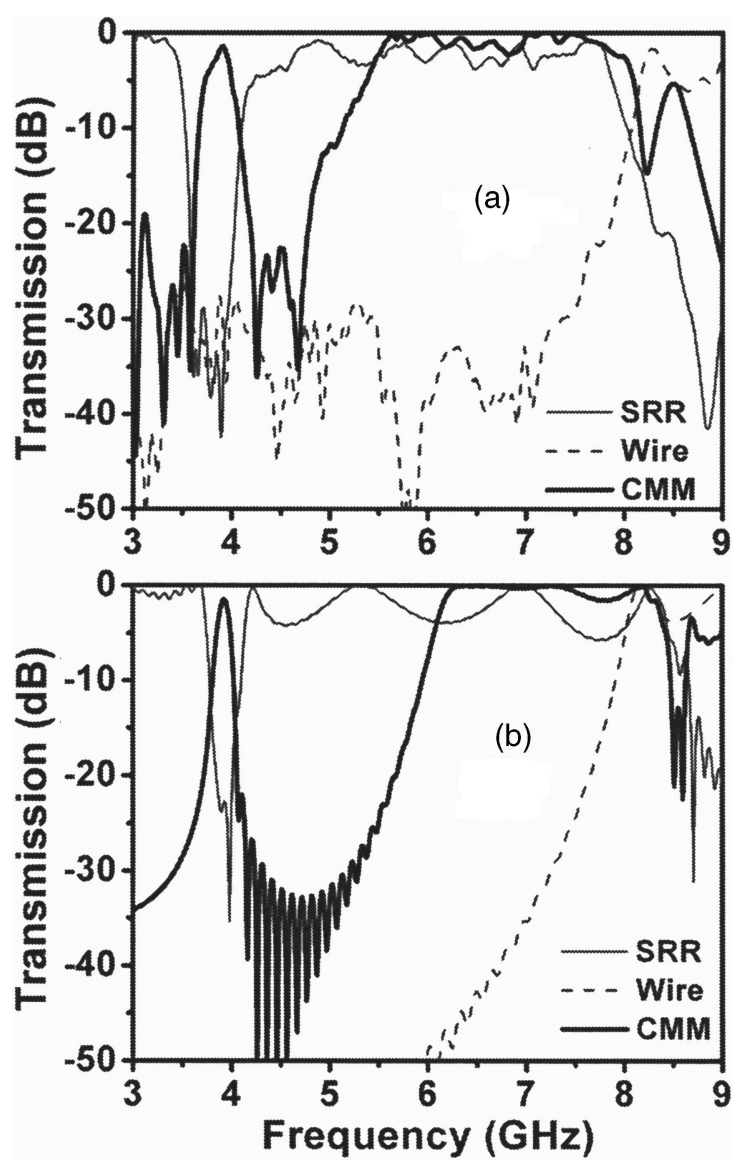

Fig. 4. Transmission spectra of SRRs, wires, and open CMMs: (a) experiment and (b) simulation.

However, as Fig. 3 suggests, the $\epsilon>0$ regime of the combined electric response of SRRs and wires starts at $5.3 \mathrm{GHz}$.

In conclusion, we have successfully demonstrated true left-handed behavior in free space with a high transmission peak. The left-handed transmission band exactly coincides with the region where both dielectric permittivity and magnetic permeability take negative values. By closing the splits of the SRRs, we experimentally verified the magnetic resonance of the SRR structures. Using this procedure, one can successfully identify the regions of negative permeability and negative permittivity of SRR structures. We also confirmed experimentally that $\omega_{p}$ of the CMM composed of SRRs and wires is lower than wires-only plasma frequency $\omega_{p}$, as is essential for the design of CMM structures and the interpretation of transmission spectra. Finally, our detailed numerical calculations agree quantitatively with the experimental results.

We thank T. Koschny and E. N. Economou for useful discussions. This work was supported by EUDALHM, by Defense Advanced Research Projects Agency contract MDA 972-01-2-0016, and by EU-METAMORPHOSE. The Ames Laboratory is operated for the U.S. Department of Energy by Iowa State University under contract W-7405-Eng-82. E. Ozbay acknowledges partial support from the Turkish Academy of Sciences. K. Aydin's e-mail address is aydin@fen.bilkent.edu.tr.

\section{References}

1. V. G. Veselago, Sov. Phys. Usp. 10, 504 (1968).

2. D. R. Smith, W. J. Padilla, D. C. Vier, S. C. Nemat-Nasser, and S. Schultz, Phys. Rev. Lett. 84, 4184 (2000).

3. R. A. Shelby, D. R. Smith, S. C. Nemat-Nasser, and S. Schultz, Appl. Phys. Lett. 78, 480 (2001).

4. J. B. Pendry, A. J. Holden, D. J. Robbins, and W. J. Stewart, J. Phys. Condens. Matter 10, 4785 (1998).

5. J. B. Pendry, A. J. Holden, D. J. Robbins, and W. J. Stewart, IEEE Trans. Microwave Theory Tech. 47, 2075 (1999).

6. M. Bayindir, K. Aydin, P. Markos, C. M. Soukoulis, and E. Ozbay, Appl. Phys. Lett. 81, 120 (2002).

7. K. Li, S. J. McLean, R. B. Greegor, C. G. Parazzoli, and M. H. Tanielian, Appl. Phys. Lett. 82, 2535 (2003).

8. E. Ozbay, K. Aydin, E. Cubukcu, and M. Bayindir, IEEE Trans. Antennas Propag. 51, 2592 (2003).

9. R. A. Shelby, D. R. Smith, and S. Schultz, Science 292, 77 (2001).

10. C. G. Parazzoli, R. B. Greegor, K. Li, B. E. Koltenbah, and M. Tanielian, Phys. Rev. Lett. 90, 107401 (2003).

11. A. A. Houck, J. B. Brock, and I. L. Chuang, Phys. Rev. Lett. 90, 137401 (2003).

12. T. Koschny, M. Kafesaki, E. N. Economou, and C. M. Soukoulis, Phys. Rev. Lett. 93, 107402 (2004). 